\title{
Zonificación altitudinal
}

\section{de Pinus patula a partir de conos y semillas en la sierra de Huayacocotla, Veracruz, México}

\author{
Altitudinal zoning of Pinus patula from cones and seeds \\ in the sierra de Huayacocotla, Veracruz, Mexico
}

\author{
Armando Aparicio-Rentería!, Héctor Viveros-Viveros ${ }^{*}$, Julia Hernández-Villa', Cuauhtémoc Sáenz-Romero², César \\ Ruiz-Montiel' y Jorge Alberto Pineda Posadas ${ }^{3}$
}

1 Universidad Veracruzana. Instituto de Investigaciones Forestales. Xalapa, Veracruz, México.

* Autor de correspondencia. heviveros@hormail.com
2 Universidad Michoacana de San Nicolás de Hidalgo. Instituto de Investigaciones sobre los Recursos Naturales. Morelia, Michoacán, México.
3 Instituto Tecnológico Superior de Perote. Perote, Veracruz, México.

\section{RESUMEN}

Las poblaciones de especies forestales con amplia distribución tienden a diferenciarse en caracteres morfológicos a través de gradientes altitudinales. Esto permite establecer zonas para el movimiento de semillas, que garanticen la adaptación y un mejor crecimiento de las plantas. El patrón de variación morfológica asociado a gradientes altitudinales es desconocido para muchas especies de pinos mexicanos. Por lo tanto, se exploró la variación morfológica altitudinal con base en el largo y ancho de conos y semillas de 70 árboles superiores de Pinus patula seleccionados en ocho poblaciones de Huayacocotla, Veracruz, para establecer zonas provisionales para el movimiento de semillas. Se realizaron análisis de varianza y de asociación con variables geográficas. Se registró variación significativa entre y dentro de poblaciones para todas las características evaluadas y correlación positiva de la altitud con los caracteres de los conos y el ancho de las semillas. Con base en la variación del largo y ancho del cono, así como el ancho de semilla, se sugiere la delimitación provisional de dos zonas altitudinales (zona I: 1850 m - 2350 m snm; zona II: 2350 m - 2850 m snm), para el movimiento de semilla y reforestación. Se sugiere que la semilla de cada zona se utilice provisionalmente en un intervalo de $\pm 250 \mathrm{~m}$ altitudinales a partir de su origen, sin considerar el efecto del cambio climático y mover altitudinalmente hacia arriba las fuentes de semillas $250 \mathrm{~m}$ de altitud considerando el cambio climático, para reacoplar al clima proyectado para el año 2030, o $500 \mathrm{~m}$ para el de 2060.

PALABRAS CLAVE: caracteres morfológicos, movimiento de semillas, reforestación, selección, variación morfológica altitudinal.

\section{ABSTRACT}

Populations of forest species with a wide distribution tend to differ in morphological traits across altitudinal gradients. This allows the provisional zoning for conducting seed movement that ensures the adaptation and the best growth of the plants. The pattern of morphological variation associated with altitudinal gradients is not known for many Mexican pine species. Therefore, the altitudinal morphological variation was explored based on the length and width of cones and seeds of 70 Pinus patula plus trees selected in eight populations of Huayacocotla, Veracruz, to establish provisional zones for seeds movement. Analysis of variance and association with geographical variables were performed. Significant variation was recorded between and within populations for all the evaluated characteristics and positive correlation of the altitude with the characters of the cones and the width of the seeds. Based on the variation of cone length and width as well as seed width, we suggest a provisional delineation of two altitudinal seed zones (zone I: $1850 \mathrm{~m}$ - 2350 $\mathrm{m}$ asl; zone II: $2350 \mathrm{~m}-2850 \mathrm{~m}$ asl), for the movement of seed and reforestation. It is suggested that the seed collected in each zone is used provisionally in a range of \pm 250 altitudinal $\mathrm{m}$ from the place of origin, without considering the effects climate change. Considering climatic change, it is suggested an altitudinal upward shift of seed sources of $250 \mathrm{~m}$ of altitude to realign to the climate projected for 2030 , or $500 \mathrm{~m}$ for the one to 2060 .

KEYWORDS: morphological characters, seed movement, reforestation, selection, altitudinal morphological variation. 


\section{INTRODUCCIÓN}

Pinus patula Schiede ex Schltdl. et Cham. es nativo de México, con distribución natural a lo largo de la Sierra Madre Oriental, en el Eje Neovolcánico Transversal y la Sierra Madre del Sur, se encuentra en latitudes aproximadas de $17^{\circ}$ a $24^{\circ} \mathrm{N}$ (Perry, 1991) y longitudes aproximadas de $92.333^{\circ}$ a $99.738^{\circ} \mathrm{O}$ (Eguiluz-Piedra, 1977; Vela, 1980); con altitudes que varían de los $1800 \mathrm{~m}$ hasta los $3100 \mathrm{~m}$ snm (Farjon, 2005), generalmente se encuentra creciendo en bosques templados-fríos y es una de las pocas especies de pino que crece en el bosque mesófilo de montaña (Velázquez-Martínez, Ángeles-Pérez, Llanderal-Ocampo, Román-Jiménez y Reyes-Hernández, 2004).

Este pino es de los más utilizados para establecer plantaciones fuera de su área de distribución natural, debido a su rápido crecimiento, buena conformación de copa, buena poda natural, fuste recto y madera de buena calidad (Nyoka, 2002). Por ejemplo, se han establecido plantaciones de la especie en países como Burundi, Camerún, Etiopia, Kenia, Madagascar, Malawi, Mozambique, Nigeria, República de Angola, República Democrática del Congo [Zaire], Ruanda, Sudáfrica, Suazilandia, Tanzania, Uganda, Zambia y República de Zimbabue (en África); China, India y República Democrática Socialista de Sri Lanka (en Asia); Australia, Fiji, Nueva Zelanda, Estado Independiente de Papúa Nueva Guinea (en Oceanía); y Argentina, Bolivia, Brasil, Colombia, Ecuador, Jamaica y Venezuela (en América) (Niembro, Vásquez y Sánchez, 2010; Nyoka, 2002; Wormald, 1975).

En especies con amplia distribución como P. patula se ha observado que existe variación morfológica dentro y entre distintas poblaciones como respuesta a la adaptación e interacción genética de cada individuo a distintos microambientes (Premoli y Brewer, 2007; Premoli, Raffaele y Mathiasen, 2007; Zúñiga, Sánchez-González y Granados, 2009), por lo anterior, dicha variación puede ser un indicador de variación genética o de plasticidad fenotípica (Chevin, Lande y Mace, 2010; Franiel y Wieski, 2005; Uribe-
Salas, Sáenz-Romero, González-Rodríguez, Telléz-Valdéz y Oyama, 2008; Zúñiga et al., 2009).

La variación genética de caracteres con valor adaptativo (p. ej. altura de planta, dinámica de elongación de la yema, producción de biomasa o tolerancia a bajas temperaturas) detectada a partir de ensayos de procedencia en vivero o campo, puede ayudar a determinar zonas de movimiento o transferencia de semillas (Sáenz-Romero, 2004). Una alternativa temporal, cuando se carece de estos ensayos, es utilizar la variación morfológica de caracteres de fácil medición que pueden tener valor adaptativo (p. ej. longitud de acícula, cono y semillas) (Uribe-Salas et al., 2008; Viveros-Viveros, Camarillo-Luna, Sáenz-Romero y Aparicio-Rentería, 2013).

Se ha encontrado variación a nivel población y familias para caracteres morfológicos de conos, semillas y acículas de P. patula (Alba-Landa, Rebolledo-Camacho y AparicioRentería, 1998; Viveros-Viveros et al., 2013), así como en el patrón de crecimiento en altura a los seis meses de edad a nivel de poblaciones (Sáenz-Romero, Ruiz-Talonia, Beaulieu, Sánchez-Vargas y Rehfeldt, 2011). Además, ya se cuenta con dos zonificaciones altitudinales de P. patula para bosques de la comunidad indígena de Ixtlán de Juárez, Oaxaca, México; una con base en la evaluación del crecimiento en altura de la planta a los 36 meses de edad establecidas en campo (Ruiz-Talonia, Sánchez-Vargas, Bayuelo-Jiménez, Lara-Cabrera y Sáenz-Romero, 2014) y otra con base en la variación morfológica de la longitud de acículas (Viveros-Viveros et al., 2013); ambas zonificaciones se realizaron obteniendo las diferencias mínimas significativas (Dms) entre poblaciones de cada variable que mostró un patrón de variación asociado a la altitud (altura de la planta y longitud de acículas, respectivamente), con dichas diferencias se obtuvieron los intervalos altitudinales. Es importante conocer si la variación morfológica se mantiene en otras zonas de distribución natural de la especie.

El estudio de la variación adaptativa a través de la variación de caracteres morfológicos funcionales permiten 
inferir la adaptación de las especies ante diferentes intensidades de selección que imponen los ambientes (Ipinza-Carmona, 1998), es por ello que se exploró utilizar caracteres morfológicos de conos y semillas de árboles de P. patula para estimar la variación adaptativa de la especie, la cual permitiera el establecimiento de una zonificación altitudinal para el movimiento de semillas.

\section{OBjetivos}

Los objetivos del presente trabajo fueron (1) determinar la variación existente en caracteres morfológicos de conos y semillas en árboles superiores de $P$. patula procedentes de ocho poblaciones de la Sierra de Huayacocotla, Ver., (2) estimar el porcentaje de la variación debida a las diferencias entre poblaciones y entre árboles dentro de una misma población, (3) establecer zonas provisionales para el movimiento de semilla a partir de la variación fenotípica de caracteres morfológicos con valor adaptativo; y (4) comparar la zonificación altitudinal resultante con las existentes para la especie en otras regiones del país.

\section{MATERIALES Y MÉTODOS}

\section{Recolección del material biológico}

Se recolectaron conos maduros y sanos de 70 árboles de $P$. patula seleccionados fenotípicamente en ocho poblaciones de la Sierra de Huayacocotla, Veracruz, México, en un intervalo altitudinal de $1860 \mathrm{~m}$ a $2730 \mathrm{~m}$ snm (Tabla 1, Fig.
1), las poblaciones estuvieron separadas en promedio por $5.5 \mathrm{~km} \mathrm{y} 145 \mathrm{~m}$ altitudinales. En cada población se seleccionaron en promedio de nueve a ocho árboles, con base en los criterios del Programa Internacional para la Conservación y Domesticación de Árboles [Camcore] (2007a), selección de individuos libres de plagas y enfermedades, con buena rectitud de fuste y de condición dominante o codominante. Para evitar en lo posible relaciones de parentesco se mantuvo una distancia superior de $100 \mathrm{~m}$ entre cada árbol seleccionado y se generó un código de identificación con la finalidad de conservar el pedigrí del material recolectado (Camcore, 2007b, 2008). La calidad de los árboles con respecto a la rectitud del fuste y poda natural se clasificó de manera subjetiva en una escala de uno, dos y tres, en la que tres indica las mejores calidades en la región de Huayacocotla, durante la selección se aceptaron únicamente ejemplares con una rectitud del fuste dos y tres. De cada árbol, se recolectaron de 70 a 200 conos maduros libres de ataques visibles de plagas y enfermedades, de acuerdo con la producción de conos que presentó cada árbol, en la recolección de conos no se consideró la rama de la que provenían, ni la exposición, tampoco se seccionó la copa del árbol para realizarla; los conos de cada árbol se mantuvieron por separado e identificados en costales de yute, para su posterior evaluación y se trasladaron al Banco Central de Germoplasma Forestal Los Molinos, Perote, Veracruz.

TABLA 1. Localización geográfica y altitudinal de ocho poblaciones de Pinus patula de la región de Huayacocotla, Veracruz, México.

\begin{tabular}{lccc}
\hline \multicolumn{1}{c}{ Población } & Latitud $(N)$ & Longitud $^{\prime}(\mathrm{O})$ & Altitud (m snm) \\
\hline La Selva & $98^{\circ} 30.984^{\prime}$ & $20^{\circ} 34.878^{\prime}$ & 1860 \\
Puerto Lobos & $98^{\circ} 26.336^{\prime}$ & $20^{\circ} 24.213^{\prime}$ & 2331 \\
Potrero Monrroy & $98^{\circ} 44.708^{\prime}$ & $20^{\circ} 24.750^{\prime}$ & 2493 \\
Acantilado & $98^{\circ} 31.224^{\prime}$ & $20^{\circ} 29.645^{\prime}$ & 2650 \\
Palo Bendito & $98^{\circ} 31.729^{\prime}$ & $20^{\circ} 30.412^{\prime}$ & 2650 \\
Canalejas-Otates & $98^{\circ} 26.132^{\prime}$ & $20^{\circ} 26.625^{\prime}$ & 2600 \\
Arroyo Prieto & $98^{\circ} 31.714^{\prime}$ & $20^{\circ} 29.135^{\prime}$ & 2300 \\
Las Jarillas & $98^{\circ} 26.049^{\prime}$ & $20^{\circ} 23.705^{\prime}$ & 2730 \\
\hline
\end{tabular}




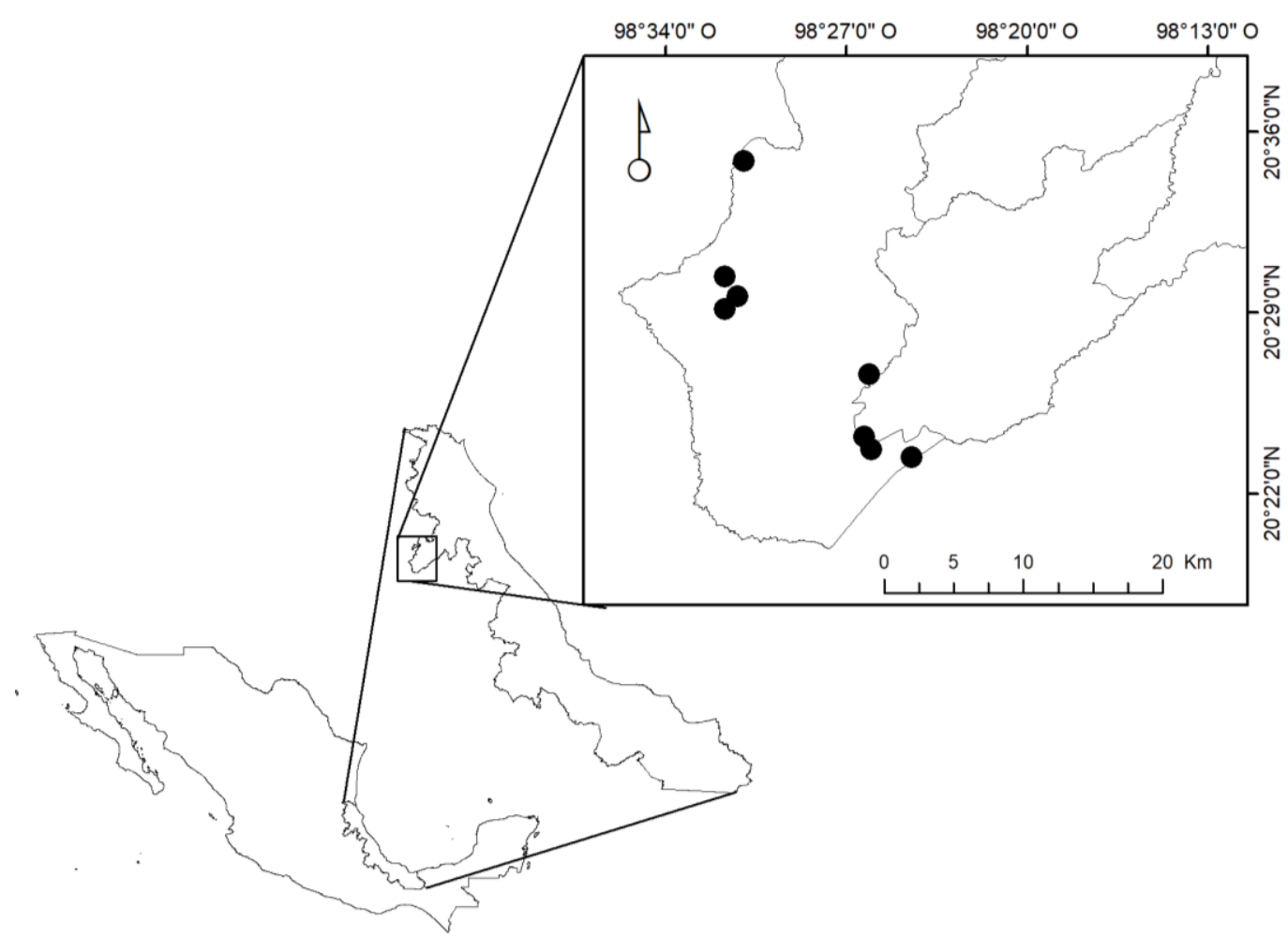

FIGURA 1. Localización geográfica de ocho poblaciones de Pinus patula en la región de Huayacocotla, Veracruz, México.

\section{Mediciones morfológicas}

Se determinó la longitud $(0.1 \mathrm{~cm})$ y el diámetro $(0.1 \mathrm{~cm})$ de una muestra aleatoria de 25 conos cerrados de cada árbol. La longitud del cono se midió desde la incisión del pedúnculo hasta el ápice del cono y el diámetro en la parte media del mismo con un vernier digital. Así mismo, de una muestra aleatoria de 50 semillas por árbol, se determinó el largo $(0.1 \mathrm{~mm})$ y ancho $(0.1 \mathrm{~mm})$ de cada una. La longitud se midió desde la base hasta la punta de la semilla y el ancho en la parte más amplia de la misma.

\section{Análisis estadístico}

Para determinar la existencia de variación significativa entre poblaciones de las características morfológicas de conos y semillas, se realizó un análisis de la varianza mediante el procedimiento Glm del paquete de análisis estadístico SAS (SAS Institute, 2004). Se consideró como una población al conjunto de árboles seleccionados en cada sitio de muestreo. El modelo utilizado para las características evaluadas fue (ver Viveros-Viveros et al., 2013):

$$
\left.\mathrm{Y}_{i j}=\mu+\text { Población }_{\mathrm{i}}+\text { Árbol(Población }\right)_{i j}+\varepsilon_{i j k}
$$

Donde:

$\mathrm{Y}_{i j}=$ valor de la $i j$-ésima observación

$\mu=$ media general

Población $_{i}=$ efecto de la $i$-ésima población

Árbol(Población $)_{i j}=$ efecto del $j$-ésimo árbol anidado en la i-ésima población

$\varepsilon_{i j k}=$ error experimental

Se estimaron los componentes de varianza asociados a cada fuente de variación y su contribución a la varianza total, mediante el procedimiento Varcomp, opción Reml de SAS (SAS Institute, 2004). 
La asociación entre las variables morfológicas evaluadas se estimó mediante el procedimiento Corr de SAS (SAS Institute, 2004).

Para determinar la existencia de un patrón de variación morfológica, expresado como la asociación entre los valores medios por población de cada característica evaluada con las variables geográficas (altitud, latitud Norte y longitud Oeste) de cada población, se utilizó el procedimiento Reg de SAS (SAS Institute, 2004) con el siguiente modelo estadístico (ver Viveros-Viveros et al., 2013):

$$
\mathrm{Y}_{i}=\mu+\beta_{0}+\beta_{1} \mathrm{X}_{i}+\varepsilon_{i}
$$

Donde:

$\mathrm{Y}_{i}=$ valor de la $i$-ésima observación

$\mu=$ media general

$\beta_{0}=$ interceptada

$\beta_{1}=$ parámetro de regresión

$\mathrm{X}_{i}=$ variable geográfica de la $i$-ésima población

$\varepsilon_{i}=$ error experimental

\section{Zonificación altitudinal}

Posterior a los análisis, para definir la zonificación altitudinal, del total de las variables evaluadas se tomaron las tres que mostraron diferencias significativas entre poblaciones y además un patrón altitudinal significativo (longitud y diámetro de conos y ancho de semilla), el cual se detectó a partir de la correlación positiva existente entre las variables y la altitud de origen de las poblaciones. Se estimaron las diferencias mínimas significativas (Dms) entre poblaciones de cada una de las tres variables antes mencionadas, con un $\alpha=0.20$, para obtener los intervalos altitudinales; cabe mencionar que el $\alpha=0.20$ implica reglas más conservadoras para el movimiento de semillas, debido a que los intervalos altitudinales resultantes (que hacen a dos poblaciones significativamente diferentes) son menores y, por tanto, habrá más restricciones para el movimiento de la semilla (Sáenz-Romero, Gúzman-Reyna y Rehfeldt, 2006). Estos valores de $\alpha$ se han usado previamente para establecer límites de las zonas altitudinales resultantes tanto de evaluar caracteres cuantitativos (Sáenz-Romero et al., 2006 y 2012; Sáenz-Romero y Tapia-Olivares, 2008; Viveros-Viveros et al., 2009) como morfológicos (UribeSalas et al., 2008; Viveros-Viveros et al., 2013).

\section{Resultados}

\section{Variación morfológica}

Todas las variables evaluadas mostraron variación significativa tanto entre poblaciones como entre árboles dentro de poblaciones $(\mathrm{P}<0.0001$, Tabla 2$)$.

TABLA 2. Media, error estándar (E.E.), porcentaje de contribución a la varianza total (\%) y significancia (P) de características morfológicas de conos y semillas de ocho poblaciones de Pinus patula en Huayacocotla, Veracruz, México.

\begin{tabular}{|c|c|c|c|c|c|c|c|}
\hline \multirow{2}{*}{ Variable } & \multirow{2}{*}{ Media } & \multirow{2}{*}{ E.E. } & \multicolumn{2}{|c|}{ Población } & \multicolumn{2}{|c|}{ Árbol(Población) } & \multirow{2}{*}{$\begin{array}{c}\text { Error } \\
\%\end{array}$} \\
\hline & & & $\%$ & $P$ & $\%$ & $P$ & \\
\hline Longitud de cono $(\mathrm{cm})$ & 7.86 & 0.18 & 12.2 & $<0.0001$ & 48.2 & $<0.0001$ & 39.6 \\
\hline Diámetro de cono $(\mathrm{cm})$ & 3.37 & 0.06 & 7.4 & $<0.0001$ & 50.2 & $<0.0001$ & 42.4 \\
\hline Largo de semilla (mm) & 4.94 & 0.06 & 0.1 & $<0.0001$ & 44.1 & $<0.0001$ & 55.8 \\
\hline Ancho de semilla (mm) & 2.55 & 0.03 & 2.8 & $<0.0001$ & 31.7 & $<0.0001$ & 65.5 \\
\hline Promedio & ------- & ----- & 5.6 & -------- & 43.6 & -------- & 50.8 \\
\hline
\end{tabular}


La contribución de las poblaciones a la varianza total varió de $0.1 \%$ a $12.2 \%$, mientras que la de los árboles dentro de poblaciones fue de $31.7 \%$ a $50.2 \%$ (Tabla 2). La variable morfológica que presentó mayor variación atribuible a las poblaciones fue la longitud del cono, mientras que la de menor aporte fue el largo de semilla. Por otra parte, las características de longitud y diámetro del cono fueron las que presentaron mayor variación atribuida a árboles dentro de poblaciones y la variable con menor aporte fue el ancho de semilla (Tabla 2). En todas las variables, la contribución a la varianza total fue mayor por parte de los árboles dentro de poblaciones que entre poblaciones (Tabla 2).

\section{Asociación entre caracteres morfológicos de conos y semillas}

En casi todas las variables evaluadas se encontraron correlaciones positivas y significativas, excepto entre la longitud de cono y el ancho de semilla (Tabla 3). Las correlaciones más altas fueron entre el largo de cono y el diámetro de cono (0.57) lo que indica que entre más largo sean los conos, estos tendrán un diámetro mayor; también entre el largo y ancho de semilla (0.48), lo que indica que entre más larga sea una semilla, ésta será también más ancha.

TABLA 3. Coeficiente de correlación de Pearson entre pares de caracteres morfológicos para conos y semillas de Pinus patula en Huayacocotla, Veracruz, México.

\begin{tabular}{llll}
\hline & LC & GC & LS \\
\hline Longitud de cono (LC) (cm) & & & \\
Diámetro de cono (GC) (cm) & $0.57^{* *}$ & & \\
Largo de semilla (LS) (mm) & $0.24^{*}$ & $0.38^{*}$ & \\
Ancho de semilla (AS) (mm) & $0.19^{\text {ns }}$ & $0.35^{*}$ & $0.48^{* *}$ \\
\hline
\end{tabular}

ns $=$ no significativa, ${ }^{*}$ significativa $\mathrm{P}<0.05,{ }^{* *}$ significativa $\mathrm{P}<0.01$

\section{Patrones altitudinales de variación morfológica}

No se encontró asociación significativa de los caracteres evaluados con la latitud y la longitud. Por otra parte, los caracteres de conos y el ancho de semilla mostraron asociación positiva significativa ( 0.52 para el largo y 0.55 para el diámetro de cono) con la altitud de origen de las poblaciones (Fig. $2 \mathrm{~A}, \mathrm{~B}$ y C). Es decir, las poblaciones de mayor altitud (a partir de $2331 \mathrm{~m} \mathrm{snm}$ ) presentaron conos con mayor diámetro y longitud, así como semillas más anchas; mientras que en las poblaciones de menor altitud los conos mostraron menor diámetro y longitud y semillas menos anchas (Fig. 2 B y C).
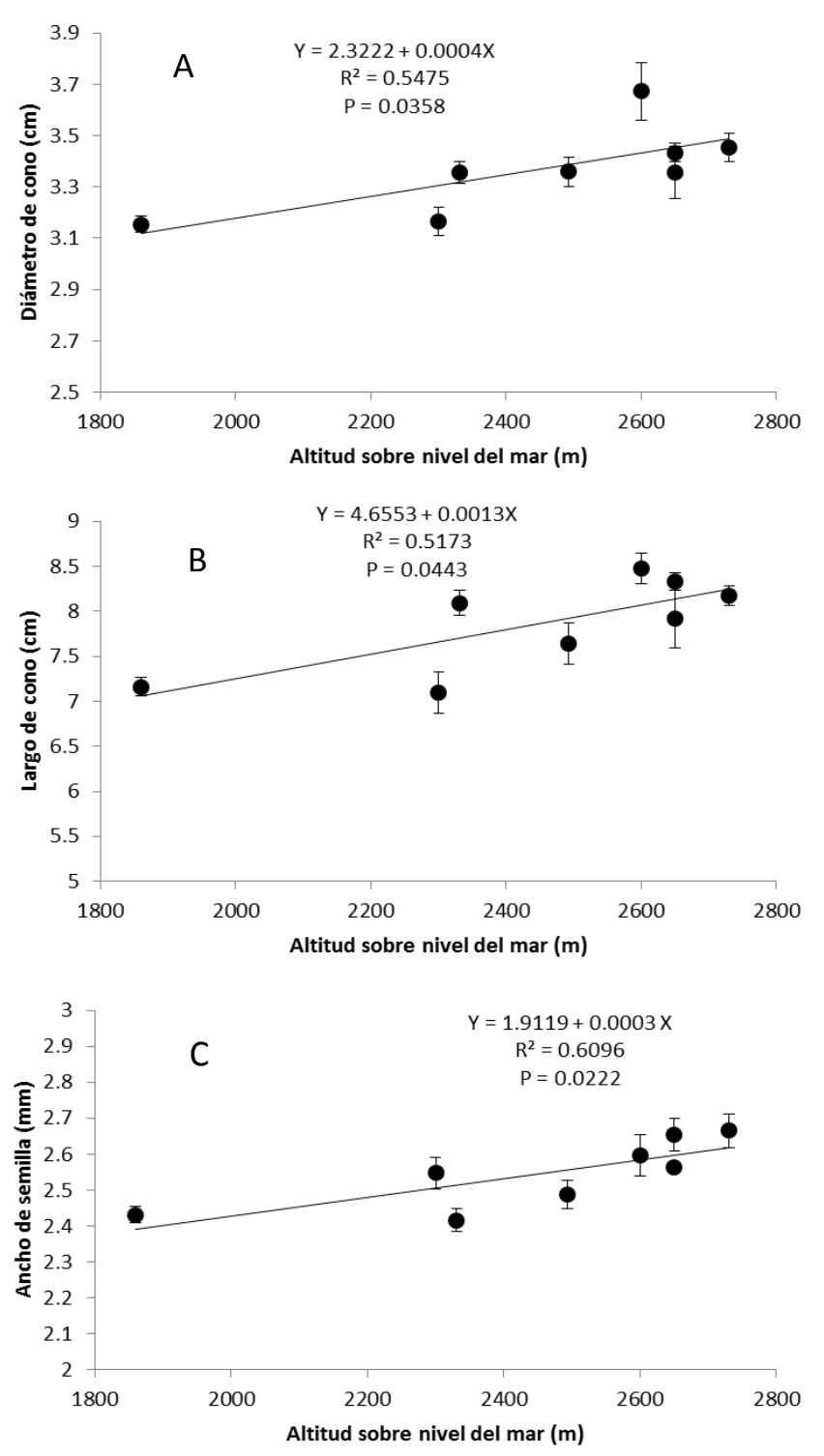

FIGURA 2. Relación entre el diámetro del cono (A), largo del cono (B) y ancho de la semilla (C) con la altitud de origen de ocho poblaciones de Pinus patula en la región de Huayacocotla, Veracruz, México.

Las barras verticales representan el intervalo de confianza al 95\%. 


\section{Zonificación altitudinal provisional}

Utilizando la diferencia mínima significativa para el largo de cono $\left(\mathrm{Dms}_{\alpha}=0.2=0.7603 \mathrm{~cm}\right)$ se estimó una amplitud de las zonas altitudinales de $500 \mathrm{~m}$, mientras que la diferencia mínima significativa para el diámetro de cono $\left(\mathrm{Dms}_{\alpha}=0.2=\right.$ $0.2469 \mathrm{~cm})$ y para el ancho de la semilla $\left(\mathrm{Dms}_{\alpha}=0.2=0.1841\right.$ $\mathrm{mm}$ ) dan una amplitud de $525 \mathrm{~m}$. Por lo anterior, se decidió delimitar la amplitud de las zonas altitudinales obteniendo un promedio de las tres amplitudes de las zonas altitudinales; obteniendo aproximadamente $517 \mathrm{~m}$ snm de diferencia altitudinal, que se redondeó a $500 \mathrm{~m}$ para fines prácticos. Con esta amplitud se establecieron y delimitaron dos zonas altitudinales para el movimiento de semillas, que cubren la totalidad del área de distribución altitudinal de $P$. patula en la región. La primera zona (parte baja de la distribución natural) se ubicó entre 1850 m y $2350 \mathrm{~m}$ snm y la segunda zona entre $2350 \mathrm{~m}$ y $2850 \mathrm{~m}$ snm (parte alta de la distribución natural).

\section{Transferencia de semillas}

Con base en las zonas altitudinales delimitadas, se sugiere que la semilla recolectada en áreas cercanas al centro de cada zona altitudinal se utilice de manera provisional en reforestaciones dentro del intervalo altitudinal de cada zona altitudinal, es decir $250 \mathrm{~m}$ hacia arriba o hacia bajo de la parte central de cada zona altitudinal, $250 \mathrm{~m}$ hacia arriba y hacia abajo dan la amplitud de cada zona altitudinal (500 $\mathrm{m})$; sin embargo si se recolecta semilla en áreas cercanas a los límites altitudinales (inferior o superior), cabe la pregunta ¿se puede mover semilla fuera de la zona altitudinal de recolección?, la respuesta es sí, siempre y cuando el movimiento se mantenga en $\pm 250 \mathrm{~m}$ altitudinales a partir del lugar de recolección (mitad de la amplitud de cada zona altitudinal), no importando si existe traslape entre zonas altitudinales.

\section{DISCUSIÓN}

\section{Variación morfológica}

Los valores promedio de los caracteres de conos y semillas encontrados en el presente trabajo están incluidos en los valores de variación reportados por Niembro et al. (2010) para la especie, de $4 \mathrm{~cm}$ a $10 \mathrm{~cm}$ para la longitud del cono, de $3 \mathrm{~cm}$ a $5 \mathrm{~cm}$ para el diámetro de cono, de $5 \mathrm{~mm}$ a $6 \mathrm{~mm}$ para el largo de la semilla y de $2 \mathrm{~mm}$ a $3 \mathrm{~mm}$ para el ancho de la semilla.

Considerando que la variación morfológica es el resultado de la expresión fenotípica, que a su vez es producto de la variación genética, la ambiental y la interacción entre ambos, es muy probable que la existencia de variación morfológica sea un indicador de la existencia de la variación genética (Ipinza-Carmona, 1998). En este caso, la variación fenotípica clinal entre poblaciones, es un indicador de la posible variación genética entre poblaciones, a lo largo de un gradiente altitudinal.

Sin embargo, la variación encontrada a nivel de poblaciones y árboles dentro de la población indica que estas características están influenciadas tanto por el ambiente como por el componente genético, sin embargo, para poder separar el efecto ambiental del genético se requiere del establecimiento de ensayos de procedencias, siendo otra alternativa de estimación de la contribución de ambos factores, pero teniendo un mayor control en la aportación genética (Ipinza-Carmona, 1998). La variación a nivel de poblaciones y árboles dentro de la población encontradas indican la capacidad adaptativa de las poblaciones (Rawat y Bakshi, 2011). La elevada contribución a la varianza total aportada por los árboles dentro de poblaciones coincide con trabajos similares para la misma especie (Alba-Landa et al., 1998; Viveros-Viveros et al., 2013), y resultados similares en conos y semillas de Pinus oocarpa Schiede (Nepamuceno-Martínez y SánchezAlpizar, 1987) y Pinus hartwegii Lindl. (Iglesias, Solís-Ramos y Viveros-Viveros, 2012) y reflejan la adaptación a la competencia o a ambientes locales muy diferenciados (Gil et al., 2002). Además, la variabilidad en la morfología de conos y semillas es un importante indicador de la especialización ecológica de la especie (Donahue y LópezUpton, 1996; Boulli, Baaziz y M'Hirit, 2001; Dangasuk y Panetsos, 2004; Wahid, González-Martínez, El Hadrami y Boulli, 2006). La variabilidad encontrada se puede aprovechar para la conservación (in situ o ex situ) y el uso 
sustentable de $P$. patula en la región (Loha, Tigabu, Teketay, Lundkvist y Fries, 2006).

\section{Asociación entre los caracteres morfológicos de conos y semillas}

En cuanto a la asociación entre las características de los conos y las semillas en pinos, se han observado asociaciones similares entre conos y semillas de Pinus sylvestris L. (Turna y Güney, 2009) y solamente entre características de conos de Pinus arizonica Engelm. (Rodríguez-Laguna y CapoArteaga, 2005). Lo que indica que en especies como $P$. patula y $P$. sylvestris el desarrollo y crecimiento del cono influye directamente en el desarrollo y crecimiento de la semilla (Turna y Güney, 2009).

\section{Patrones altitudinales de variación morfológica}

La asociación positiva entre la altitud de origen de las poblaciones con los caracteres de conos y el ancho de semilla indica que la presencia de conos de mayores dimensiones pudiera brindar una mayor protección de las semillas contra factores ambientales (Beaufait, 1960; Dawson, Vincent y Rocca, 1997; Linhart, 1978), entre estos factores están las heladas, las cuales son más frecuentes en las mayores elevaciones (Viveros-Viveros et al., 2013). Un patrón idéntico se reportó para el diámetro de cono en poblaciones de $P$. patula en la región de Ixtlán de Juárez en Oaxaca (Viveros-Viveros et al., 2013). La asociación positiva del tamaño del cono y el ancho de la semilla con la altitud parece un patrón atribuible a la especie $y$, es un patrón opuesto a los reportado en otras especies, por ejemplo, se reporta que, a mayor altitud, usualmente se encuentran conos, acículas y semillas más pequeñas (SáenzRomero et al., 2012). El patrón de variación encontrado para la especie en el presente trabajo es debido a que las estructuras que sirven como protección a partes vitales de los árboles, como las vainas para las acículas y los conos para las semillas, aumentan de tamaño conforme aumenta la altitud, lo anterior porque presentan mayor área para proteger a las acículas y las semillas, respectivamente de factores ambientales adversos (Niembro, 1986; Silva-Sáenz et al., 1991; Viveros-Viveros et al., 2013).

\section{Zonificación altitudinal provisional}

Viveros-Viveros et al., (2013), basados en la longitud de acículas, proponen dos zonas provisionales (zona I: $2150 \mathrm{~m}$ - 2600 m snm y zona II: $2600 \mathrm{~m}$ - $3050 \mathrm{~m}$ snm) para el movimiento de semillas de $P$. patula, aunque dichas zonas altitudinales fueron para la región de Ixtlán, Oaxaca, cada zona con una amplitud de $450 \mathrm{~m}$ snm muy semejante a lo encontrado en el presente estudio para la especie en la zona de Huayacocotla, que fue de $500 \mathrm{~m}$ snm. En contraste, ambas zonificaciones, resultaron más amplias a la realizada por Ruiz-Talonia et al., (2014) para la especie en la misma zona de Ixtlán, pero basada en la variación genética altitudinal del crecimiento en altura de la planta a los 36 meses de edad, detectada en un ensayo de procedencias en campo; en ese trabajo, se establecieron cuatro zonas (zona I: $2300 \mathrm{~m}$ - $2500 \mathrm{~m}$ snm, zona II: $2500 \mathrm{~m}-2700 \mathrm{~m}$ snm, zona III: $2700 \mathrm{~m}-2900 \mathrm{~m}$ snm y zona IV: $2900 \mathrm{~m}-3100$ $\mathrm{m}$ snm) cada una de ellas con una amplitud de $200 \mathrm{~m}$ snm. En Abies religiosa (Kunth) Schltdl. \& Cham. se ha sugerido el establecimiento de tres zonas altitudinales provisionales (zona I: $2800 \mathrm{~m}$ - $3050 \mathrm{~m}$ snm, zona II: $3050 \mathrm{~m}$ - $3300 \mathrm{~m}$ snm y zona III: $3300 \mathrm{~m}-3550 \mathrm{~m}$ snm) para el movimiento de semillas con base en la longitud de acícula y largo de cono, cada una de las zonas con una amplitud de $250 \mathrm{~m}$ altitudinales (Castellanos-Acuña, Lindig-Cisneros, SilvaFarias \& Sáenz-Romero, 2014).

\section{Transferencia de semillas}

El movimiento de semillas sugerido en el presente trabajo fue considerando las condiciones climáticas en las que se han desarrollado los individuos de cada población. La zonificación altitudinal permitió establecer reglas del movimiento de semillas más conservadoras, al sugerir realizar reforestaciones en cada zona altitudinal con planta originada de semillas recolectadas en la misma zona, con esto se corren riegos mínimos de no adaptación de las 
plantas a las condiciones climáticas de las zonas de reforestación; sin embargo cuando los objetivos son conservar las poblaciones fuera de su zona de distribución altitudinal, aumentar la productividad de la especie moviendo planta fuera de su área de distribución o contrarrestar el efecto del cambio climático, se tienen que tomar decisiones más arriesgas, ante esta situación es recomendable realizar el movimiento \pm la mitad de ancho de las zonas altitudinales ( $250 \mathrm{~m}$ para el presente estudio) a partir del lugar de recolección de semillas (Sáenz-Romero et al., 2006), el argumento de esto último es que la temperatura disminuye $0.5{ }^{\circ} \mathrm{C}$ por cada $100 \mathrm{~m}$ snm de movimiento altitudinal hacia arriba (Sáenz-Romero et al., 2010), ante esta situación existe una mayor probabilidad de que las plantas resistan una disminución de la temperatura de $1.5{ }^{\circ} \mathrm{C}$ (movimiento de $250 \mathrm{~m}$ ) a una de $2.5{ }^{\circ} \mathrm{C}$ (movimiento de 500 m) (Sáenz-Romero y Tapia-Olivares, 2008). Ante el escenario de un aumento de la temperatura, se podría adoptar la siguiente regla: recolectar en la zona altitudinal I (de $1850 \mathrm{~m}$ a $2350 \mathrm{~m} \mathrm{snm}$ ), producir planta en vivero y reforestar la zona inmediata superior (zona II: de $2350 \mathrm{~m}$ y $2850 \mathrm{~m} \mathrm{snm}$ ). Esto representaría un movimiento altitudinal máximo de $500 \mathrm{~m}$ snm. Con ello, se acoplarían los genotipos al clima esperado para la década centrada en el año 2060 , que se ha proyectado será $2.3^{\circ} \mathrm{C}$ más cálido. Teniendo en cuenta la consideración antes mencionada de que la temperatura disminuye $0.5^{\circ} \mathrm{C}$ por cada $100 \mathrm{~m} \mathrm{snm}$ de movimiento altitudinal hacia arriba (Sáenz-Romero et al., 2010), un movimiento de $500 \mathrm{~m}$ altitudinalmente hacia arriba representa una compensación de $2.5^{\circ} \mathrm{C}$, muy cercano a los $2.3^{\circ} \mathrm{C}$. Este movimiento no está exento de riesgos. Es de esperarse que ocurrirán ciertos daños por heladas (Sáenz-Romero y Tapia-Olivares, 2008). Un lineamiento menos arriesgado sería algo más cercano a lo sugerido por Ruiz-Talonia et al. (2014), quien, con base en la magnitud de la diferenciación genética entre poblaciones para caracteres cuantitativos, recomiendan para la especie mover hacia arriba $200 \mathrm{~m}$ altitudinales a partir del sitio de recolección, para de manera aproximada reacoplarse al clima predicho para el año 2030, para dicho año se espera un aumento de temperatura de $1.5^{\circ} \mathrm{C}$. Por ello, sería posible mover altitudinalmente hacia arriba la mitad del ancho de zona altitudinal, $250 \mathrm{~m}$, a partir de la zona de recolección.

\section{CONCLUSIONES}

La diferenciación morfológica entre poblaciones de $P$. patula fue significativa en la región de Huayacocotla, Veracruz. El patrón de variación encontrado muestra correlación positiva entre la altitud de origen de las poblaciones con los caracteres de cono y el ancho de la semilla. Es decir, las poblaciones de mayor altitud mostraron conos más anchos y largos, así como semillas más anchas, lo cual es un patrón inusual. De acuerdo con la variación fenotípica de caracteres morfológicos se delimitaron dos zonas altitudinales para el movimiento de semillas, cada una con una amplitud de $500 \mathrm{~m}$ snm. Sin considerar el cambio climático, es posible reforestar cada zona con planta originada a partir de semilla de la misma zona, o bien mover planta $\pm 250 \mathrm{~m}$ snm del sitio de recolecta. Considerando el cambio climático, el movimiento de semilla tendría que ser $250 \mathrm{~m}$ snm hacia arriba para reacoplar al clima proyectado para la década centrada en el año 2030, o de $500 \mathrm{~m}$ snm y así poder reacoplar al año 2060. Sin embargo, esta zonificación altitudinal basada en caracteres morfológicos, al igual que las que anteriormente se han realizado, tiene un carácter provisional y su implementación se recomienda en los casos en los que aún no se han establecido ensayos de procedencias para evaluar la variación genética de caracteres cuantitativos.

\section{REFERENCIAS}

Alba-Landa, J., Rebolledo-Camacho, V., \& Aparicio-Rentería, A. (1998). Variación morfométrica en conos y semillas de Pinus patula Schlect. et Cham. de Huayacocotla, Veracruz, México. Foresta Veracruzana, 1(1), 37-42.

Beaufait, W. R. (1960). Some effects of high temperatures on the cones and seeds of jack pine. Forest Science, 6(3), 194-199. doi: 10.1093/forestscience/6.3.194.

Boulli, A., Baaziz, M., \& M'Hirit, O. (2001). Polymorphism of natural populations of Pinus halepensis Mill. in Morocco as revealed by morphological characters. Euphytica, 119, 309-316. doi: 10.1023/A:1017571904517 
Castellanos-Acuña, D., Lindig-Cisneros, R. A., Silva-Farias, M. Á., \& Sáenz-Romero, C. (2014). Zonificación altitudinal provisional de Abies religiosa en un área cercana a la reserva de la biósfera de la mariposa monarca, Michoacán. Revista Chapingo Serie Ciencias Forestales y del Ambiente, 20(2), 215-225. doi: 10.5154/r.rchscfa.2013.11.041

Chevin, L. M., Lande, R., \& Mace, G. M. (2010). Adaptation, plasticity, and extinction in a Changing environment: Towards a predictive theory. PLoS Biology, 8(4), 1-8. doi: 10.1371/journal.pbio.1000357

Dangasuk, O. G. \& Panetsos, K. P. (2004). Altitudinal and longitudinal variations in Pinus brutia (Ten.) of Crete Island, Greece: some needle, cone and seed traits under natural habitats. New Forests, 27(3), 269-284. doi: 10.1023/B:NEFO.000

Dawson, C., Vincent, J. F. V., \& Rocca, A. M. (1997). How pine cones open. Nature, 390 (6661), 668. doi: $10.1038 / 37745$

Donahue, J. K. \& López-Upton, J. (1996). Geographical variation in leaves, cone and seeds of Pinus gregii in native forests. Forest Ecology and Management, 82(1-3), 145-157. doi: 10.1016/03781127(95)03677-6

Eguiluz-Piedra, T. (1977). Los pinos del mundo. Chapingo, México: Escuela Nacional de Agricultura. Departamento de Enseñanza, Investigación y Servicio en Bosques.

Farjon, A. (2005). Pines. Drawings and descriptions of the genus Pinus (2da. ed.). Boston, Estados Unidos: Brill.

Franiel, I. \& Wieski, K. (2005). Leaf features of silver birch (Betula pendula Roth). variability within and between two populations (uncontaminated $v \mathrm{sb}$-contaminated and $\mathrm{Zn}$-contaminated site). Trees, 19(1), 81-88. doi: 10.1007/s00468-004-0366-3

Gil, L., Climent, J., Nanos, N., Mutke, S., Ortiz, I., \& Schiller, G. (2002). Cone morphology variation in Pinus canariensis Sm. Plant Systematics and Evolution 235(1-4), 35-51. doi: 10.1007/s00606-002-0218-9

Iglesias, L. G., Solís-Ramos, L. Y., \& Viveros-Viveros, H. (2012). Variación morfométrica en dos poblaciones naturales de Pinus hartwegii Lindl. del estado de Veracruz. Pyton, 81(2), 239-246.

Ipinza-Carmona, R. (1998). Ciclo de mejoramiento genético. En R. Ipinza, B. Gutiérrez \& V. Emhart (Eds.), Curso mejora genética forestal operativa (pp. 49-64). Valdivia, Chile: Universidad Austral de Chile.

Linhart, Y. B. (1978). Maintenance of variation in cone morphology in California closed-cone pines; the roles of fire, squirrels and seed output. The Southwestern Naturalist, 23(1), 29-40. doi: $10.2307 / 3669977$

Loha, A., Tigabu, M., Teketay, D., Lundkvist, K., \& Fries, A. (2006). Provenance variation in seed morphometric traits, germination, and seedling growth of Cordia africana Lam. New Forests, 32,71-86. doi: 10.1007/s11056-005-3872-2

Nepamuceno-Martínez, F. \& Sánchez-Alpizar V. (1987). Variaciones morfológicas en 10 poblaciones de Pinus oocarpa Schiede. Revista Ciencia Forestal, 12(61), 3-17.

Niembro R., A. (1986). Mecanismo de reproducción sexual en pinos. México: Limusa.

Niembro R., A., Vásquez T., M., \& Sánchez S., O. (2010). Árboles de Veracruq: 100 especies para la reforestación estratégica. Veracruz, México: Gobierno del Estado de Veracruz. Universidad Veracruzana.

Nyoka, B. I. (2002). Pinus patula Schiede ex Schltdl. \& Cham. En CAB International (Comp.), Pines of silvicultural importance. London, UK: CABI Publising.

Perry, P. J. (1991). The pines of Mexico and Central America. Portland, Oregon: Timber Press.

Premoli, A. C. \& Brewer, C. A. (2007). Environmental vs. genetically driven variation in ecophysiological traits of Nothofagus pumilio from contrasting elevations. Australian Journal of Botany, 55(6), 585591. doi: 10.1071/BT06026

Premoli, A. C., Raffaele, E., \& Mathiasen, P. (2007). Morphological and phenological differences in Nothofagus pumilio from contrasting elevations. Austral Ecology, 32(5), 515-523. doi: 10.1111/j.14429993.2007.01720.x

Programa Internacional para la Conservación y Domesticación de Árboles [Camcore] (2007a). Objetivos y resultados del programa Camcore. Boletin de noticias Camcore para México y Centroamérica, 1(1), 4.

Programa Internacional para la Conservación y Domesticación de Árboles [Camcore] (2007b). Conservación de especies y procedencias en Camcore- ¿Qué conservamos y ensayamos? Boletín de noticias Camcore para México y Centroamérica, 1(4), 5-6.

Programa Internacional para la Conservación y Domesticación de Árboles [Camcore]. (2008). Colectas, procesamiento y almacenamiento de semillas. Boletín de noticias Camcore para México y Centroamérica, 2(1), 3 .

Rawat, K. \& Bakshi, M. (2011). Provenance variation in cone, seed and seedling characteristics in natural populations of Pinus wallichiana A.B. Jacks (Blue Pine) in India. Annals of Forest Research, 54(1), 3955.

Rodríguez-Laguna, R. \& Capo-Arteaga, M. A. (2005). Morfología de acículas y conos en poblaciones naturales de Pinus arizonica Engelm. Ra Ximhai, 1(1), 131-152. 
Ruiz-Talonia, L. F., Sánchez-Vargas, N. M., Bayuelo-Jiménez, J. S., LaraCabrera, S. I. \& Sáenz-Romero, C. (2014). Altitudinal genetic variation among native Pinus patula provenances: performance in two locations, seed zone delineation and adaptation to climate change. Silvae Genetica, 63(4), 139-149. doi: 10.1515/sg-2014-0019

Sáenz-Romero, C. (2004). Zonificación estatal y altitudinal para la colecta y movimiento de semillas de coníferas en México. En J.J. VargasHernández, B. Bermejo-Velázquez \& F.T. Ledig (Eds.), Manejo de recursos genéticos forestales (p. 72-86). México: Conafor-Comisión Forestal de América del Norte.

Sáenz-Romero, C., Gúzman-Reyna, R. R., \& Rehfeldt, G. R. (2006). Altitudinal genetic variation among Pinus oocarpa populations in Michoacán, México. Implications for seed zoning, conservation, tree breeding and global warming. Forest Ecology and Management, 229(1-3), 340-350. doi: 10.1016/i.foreco.2006.04.014

Sáenz-Romero, C. \& Tapia-Olivares, B. L. 2008. Genetic variation in frost damage and seed zone delineation within an altitudinal transect of Pinus devoniana (P. michoacana) in Mexico. Silvae Genetica, 57(3), 165-170. doi: 10.1515/sg-2008-0025

Sáenz-Romero, C., Rehfeldt, G. R., Crookston, N. L., Duval, P., StAmant, R., Beaulieu, J., \& Richardson, B. A. 2010. Spline models of contemporary, 2030, 2060 and 2090 climates of Mexico and their use in understanding climate-change impacts on the vegetation. Climatic Change, 102(3-4), 595-623. doi: 10.1007/s10584-009-9753-5

Sáenz-Romero, C., Ruiz-Talonia, L. F., Beaulieu, J., Sánchez-Vargas, N. M., \& Rehfeldt, G. R. 2011. Genetic variation among Pinus patula populations along an altitudinal gradient. Two environment nursery tests. Revista Fitotecnia Mexicana, 34(1), 19-25.

Sáenz-Romero, C., Aguilar-Aguilar, S., Silva-Farías, M. A., MadrigalSánchez, X., Lara- Cabrera, S., \& López-Upton, J. 2012. Altitudinal morphological variation among Pinus devoniana Lindl. (=P. michoacana Martínez) populations and putative variety cornuta in central-west México. Revista Mexicana de Ciencias Forestales, 3(13), 17-28.

Statistical Analysis System Institute [SAS Institute]. 2004. SAS/STAT 9.1 User's Guide. Cary, N.C.: SAS Institute Inc.

Silva-Sáenz, P., Ortega-Rodríguez, J. M., Montero-Castro, J. C., Hernández-Valencia, F., Robles del Valle, J. S., \& RodríguezLozano, G. 2011. Manual de Prácticas de Laboratorio de Botánica II. Ciclo 2011. Pteridofitas y Gimnospermas. Morelia, Michoacán, México: Facultad de Biología. Universidad Michoacana de San Nicolás de Hidalgo.
Turna, I. \& Güney, D. 2009. Altitudinal variation of some morphological characters of Scots pine (Pinus sylvestris L.) in Turkey. African Journal of Biotechnology, 8(2), 202-208.

Uribe-Salas, D., Sáenz-Romero, C., González-Rodríguez, A., TellézValdéz, O., \& Oyama, K. 2008. Foliar morphological variation in the white oak Quercus rugosa Née (Fagaceae) along a latitudinal gradient in Mexico: Potential implications for management and conservation. Forest Ecology and Management, 256(12), 2121-2126. doi: $10.1016 /$ i.foreco.2008.08.002

Vela G., L. 1980. Contribución a la Ecología de Pinus patula Schl. et Cham. México, D.F.: INIF. SARH.

Velázquez-Martínez, A., Ángeles-Pérez, G., Llanderal-Ocampo, T., Román-Jiménez, A. R., \& Reyes-Hernández, V. 2004. Monografía de Pinus patula. Zapopan, Jalisco, México: Semarnat. Conafor. Colegio de Postgraduados.

Viveros-Viveros, H., Sáenz-Romero, C., Vargas-Hernández, J. J., LópezUpton, J., Ramírez-Valverde, G., \& Santacruz-Varela, A. 2009. Altitudinal genetic variation in Pinus hartwegii Lindl. I: Height growth, shoot phenology, and frost damage in seedlings. Forest Ecology and Management, 257(3), 836-842. doi: $\underline{10.1016 / \text { j.foreco.2008.10.021 }}$

Viveros-Viveros, H., Camarillo-Luna, A. R., Sáenz-Romero, C., \& Aparicio-Rentería, A. 2013. Variación altitudinal en caracteres morfológicos de Pinus patula en el estado de Oaxaca (México) y su uso en la zonificación. Bosque, 34(2), 173-179. doi: 10.4067/S0717-92002013000200006

Wahid, N., González-Martínez, S. C., El Hadrami, I., \& Boulli, A. 2006.Variation of morphological traits in natural populations of maritime pine (Pinus pinaster Ait.) in Morocco. Annals of Forest Science, 63: 83-92. doi: 10.1051/forest:20050100

Wormald, T.J. 1975. Pinus patula. Tropical Forestry Papers No. 7. Formerly Fast Growing Timber Trees of Lowland Tropics. Oxford, England: Unit of Tropical Silviculture. Department of Forestry.

Zúñiga E., Á., Sánchez-González, A., \& Granados S., D. 2009. Análisis de la variación morfológica foliar en Quercus laeta Liebm. en el parque nacional Los Mármoles, Hidalgo, México. Revista Chapingo Serie Ciencias Forestales y del Ambiente, 15(2), 87-93.

\section{Manuscrito recibido el 7 de mayo de 2019}

Aceptado el 21 de noviembre de 2019

Publicado el 23 de abril de 2020 
Este documento se debe citar como:

Aparicio-Rentería, A., Viveros-Viveros, H., Hernández-Villa, J., Sáenz-Romero, C., Ruiz-Montiel, C., \& Pineda P., J. A. (2020). Zonificación altitudinal de Pinus patula a partir de conos y semillas en la sierra de Huayacocotla, Veracruz, México. Madera y Bosques, 26(2), e2621962. doi: 10.21829/ myb.2020.2621962

Madera y Bosques por Instituto de Ecología, A.C. se distribuye bajo una Licencia Creative Commons Atribución-NoComercialCompartirlgual 4.0 Internacional. 